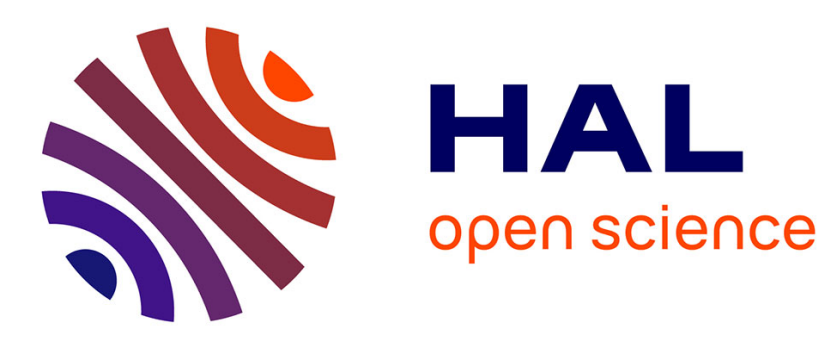

\title{
Uniform Estimation of a Constant Issued from a Fluid-Structure Interaction Problem
}

\author{
Andrei Halanay, Cornel Marius Murea
}

\section{To cite this version:}

Andrei Halanay, Cornel Marius Murea. Uniform Estimation of a Constant Issued from a FluidStructure Interaction Problem. 27th IFIP Conference on System Modeling and Optimization (CSMO), Jun 2015, Sophia Antipolis, France. pp.292-301, 10.1007/978-3-319-55795-3_27 . hal-01626880

\section{HAL Id: hal-01626880 \\ https://hal.inria.fr/hal-01626880}

Submitted on 31 Oct 2017

HAL is a multi-disciplinary open access archive for the deposit and dissemination of scientific research documents, whether they are published or not. The documents may come from teaching and research institutions in France or abroad, or from public or private research centers.
L'archive ouverte pluridisciplinaire HAL, est destinée au dépôt et à la diffusion de documents scientifiques de niveau recherche, publiés ou non, émanant des établissements d'enseignement et de recherche français ou étrangers, des laboratoires publics ou privés. 


\title{
Uniform Estimation of a Constant Issued from a Fluid-Structure Interaction Problem
}

\author{
Andrei Halanay $^{1}$, Cornel Marius Murea ${ }^{2}$ \\ 1 Department of Mathematics 1, University Politehnica of Bucharest, \\ 313 Splaiul Independenţei, RO-060042, Bucharest, Romania \\ halanay@mathem. pub.ro \\ 2 Laboratoire de Mathématiques, Informatique et Applications, \\ Université de Haute Alsace, \\ 4-6, rue des Frères Lumière, 68093 Mulhouse Cedex, France \\ cornel.murea@uha.fr
}

\begin{abstract}
We prove that the domain obtained by small perturbation of a Lipschtz domain is the union of a star-shaped domains with respect to every point of balls, such that the radius of the balls is independent of the perturbation. This result is useful in order to get uniform estimation for a fluid-structure interaction problem.
\end{abstract}

Keywords: star-shaped domains, fluid-structure interaction, fictitious domain

\section{Introduction}

In [4] and [5] the existence of a solution is studied for an elastic structure immersed in an incompressible fluid.

Let $D \subset \mathbb{R}^{2}$ be a bounded open domain. We denote by $\Omega_{0}^{S}$ the undeformed structure domain and by $\mathbf{u}=\left(u_{1}, u_{2}\right): \bar{\Omega}_{0}^{S} \rightarrow \mathbb{R}^{2}$ its displacement. A particle of the structure with initial position at the point $\mathbf{X}$ will occupy the position $\mathbf{x}=\Phi(\mathbf{X})=\mathbf{X}+\mathbf{u}(\mathbf{X})$ in the deformed domain $\bar{\Omega}_{u}^{S}=\Phi\left(\bar{\Omega}_{0}^{S}\right)$. In [5], we have assumed that $\partial \Omega_{u}^{S}$ has the uniform cone property and the geometry of the cone is independent of $\mathbf{u}$. The fluid occupies the domain $\Omega_{u}^{F}=D \backslash \bar{\Omega}_{u}^{S}$, see Figure 1 .

It is possible to construct an uniform extension operator $E$ from $\{\mathbf{v} \in$ $\left(H^{1}\left(\Omega_{u}^{S}\right)\right)^{2} ; \nabla \cdot \mathbf{v}=0$ in $\left.\Omega_{u}^{S}\right\}$ to $\left(H_{0}^{1}(D)\right)^{2}$ such that

$$
\begin{aligned}
\nabla \cdot E(\mathbf{v}) & =0, \quad \text { in } D \\
E(\mathbf{v}) & =\mathbf{v}, \quad \text { in } \Omega_{u}^{S} \\
\|E(\mathbf{v})\|_{1, D} & \leq K\|\mathbf{v}\|_{1, \Omega_{u}^{S}}
\end{aligned}
$$

where the constant $K>0$ is independent of $\Omega_{u}^{S}$, but it depends on the geometry of the cone. 


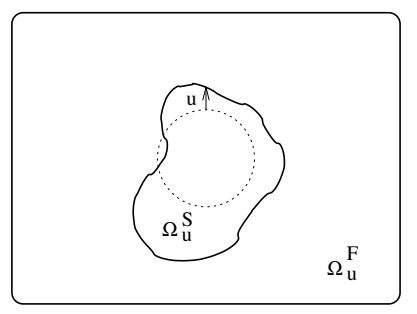

D

Fig. 1. Fluid and structure domains.

This construction is obtained by solving the Bogowskii problem in $\Omega_{u}^{F}$, see for example [3], Lemma 3.1, page 121. There exists $\mathbf{w} \in\left(H_{0}^{1}\left(\Omega_{u}^{F}\right)\right)^{2}$ such that

$$
\begin{aligned}
& \nabla \cdot \mathbf{w}=\mathbf{f}, \text { in } \Omega_{u}^{F} \\
& \mathbf{w}=0, \text { on } \partial D \\
& \mathbf{w}=0, \text { on } \partial \Omega_{u}^{S} \\
&|\mathbf{w}|_{1, \Omega_{u}^{F}} \leq K_{1}\|\mathbf{f}\|_{0, \Omega_{u}^{F}}
\end{aligned}
$$

where $\int_{\Omega_{u}^{F}} \mathbf{f} d \mathbf{x}=0$. If $\Omega_{u}^{F}$ is star-shaped with respect to any point of a ball of radius $R_{u}$, we have the following estimation of $K_{1}>0$ :

$$
K_{1} \leq c_{0}\left(\frac{\operatorname{diam}\left(\Omega_{u}^{F}\right)}{R_{u}}\right)^{2}\left(1+\frac{\operatorname{diam}\left(\Omega_{u}^{F}\right)}{R_{u}}\right)
$$

where $\operatorname{diam}\left(\Omega_{u}^{F}\right)$ is the diameter of $\Omega_{u}^{F}$. A similar result holds if the domain $\Omega_{u}^{F}$ is union of star-shaped domains with respect to any point of a ball, see Theorem 3.1, p. 129, [3]. We have $\operatorname{diam}\left(\Omega_{u}^{F}\right) \leq \operatorname{diam}(D)$, for all $\mathbf{u}$ such that $\overline{\Omega_{u}^{F}} \subset D$.

The aim of this paper is to prove that, under some geometrical assumptions, one can choose $R_{u}=R=$ constant, for small $\mathbf{u}$.

\section{Small Perturbation of the Boundary of a Star-Shaped Domain with Respect to any Point of a Ball}

We denote by $B_{R}\left(\mathbf{x}_{0}\right)$ the open ball of radius $R$ centered at $\mathbf{x}_{0}$, i.e. $B_{R}\left(\mathbf{x}_{0}\right)=$ $\left\{\mathbf{x} \in \mathbb{R}^{2} ;\left|\mathbf{x}_{0}-\mathbf{x}\right|<R\right\}$, where $|\cdot|$ is the euclidean norm. A domain $\Omega$ is starshaped with respect to every point of a ball $B_{R}\left(x_{0}\right)$ such that $\bar{B}_{R}\left(x_{0}\right) \subset \Omega$, if and only if, for every $\mathbf{x} \in B_{R}\left(x_{0}\right)$ and $\mathbf{y} \in \Omega$, the segment with ends $\mathbf{x}, \mathbf{y}$ is included in $\Omega$. A characterization of such a domain is that every ray starting from a point $\mathbf{x} \in B_{R}\left(x_{0}\right)$ intersects the boundary of the domain at only one point. 


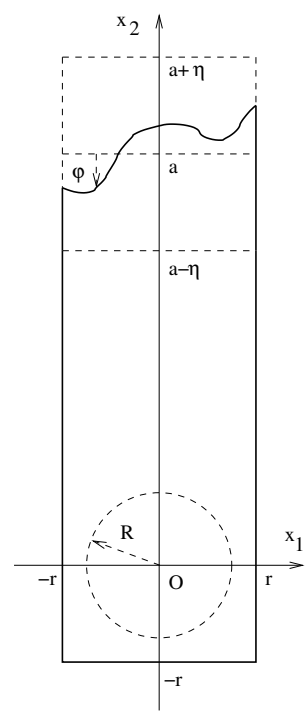

Fig. 2. A star-shaped domain with respect to every point of a ball.

Proposition 1. Let $a, r>0$ be two constants such that

$$
a \geq 4 r
$$

and $\varphi \in W^{1, \infty}(-r, r)$ be such that

$$
\begin{gathered}
\exists \eta \in(0, r), \quad \sup _{x \in(-r, r)}|\varphi(x)| \leq \eta \\
\exists L>0, \quad \sup _{x \in(-r, r)}\left|\varphi^{\prime}(x)\right| \leq L .
\end{gathered}
$$

We define the domain, see Figure 2

$$
\Omega_{\varphi}=\left\{\left(x_{1}, x_{2}\right) \in \mathbb{R}^{2} ;-r<x_{1}<r,-r<x_{2}<a+\varphi\left(x_{1}\right)\right\} .
$$

Let $R \in(0, r)$. If $L<\frac{a-2 r}{2 r}$, then the domain $\Omega_{\varphi}$ is star-shaped with respect to every point of the ball $B_{R}(0)$.

Proof. Since $R<r$, then $\bar{B}_{R}(0) \subset \Omega_{\varphi}$. Let $\mathbf{x} \in B_{R}(0)$. We suppose that a ray starting from this point cuts $\partial \Omega_{\varphi}$ in two points $\mathbf{y}$ and $\mathbf{z}$ and $\mathbf{y} \in(\mathbf{x}, \mathbf{z})$, i.e. $\mathbf{y}$ is on the segment with ends $\mathbf{x}$ and $\mathbf{z}$.

Let us denote the top boundary of $\Omega_{\varphi}$ by

$$
\Gamma_{\varphi}=\left\{\left(x_{1}, a+\varphi\left(x_{1}\right)\right) \in \mathbb{R}^{2} ;-r<x_{1}<r\right\} .
$$

If $\mathbf{y}, \mathbf{z} \in \partial \Omega_{\varphi} \backslash \Gamma_{\varphi}$ it follows that the ray cuts the boundary of the strip

$$
C=\left\{\left(x_{1}, x_{2}\right) \in \mathbb{R}^{2} ;-r<x_{1}<r,-r<x_{2}\right\},
$$


twice, which is not true because the $C$ is a convex set and a convex set is starshaped with respect to every interior point.

If we have only $\mathbf{y} \in \partial \Omega_{\varphi} \backslash \Gamma_{\varphi}$, then $\mathbf{z}$ belongs to the exterior of the strip $C$, consequently $\mathbf{z} \notin \Gamma_{\varphi}$.

So, we will study only two cases:

i) $\mathbf{y}, \mathbf{z} \in \Gamma_{\varphi}$;

ii) $\mathbf{y} \in \Gamma_{\varphi}$ and $\mathbf{z} \in \partial \Omega_{\varphi} \backslash \Gamma_{\varphi}$.
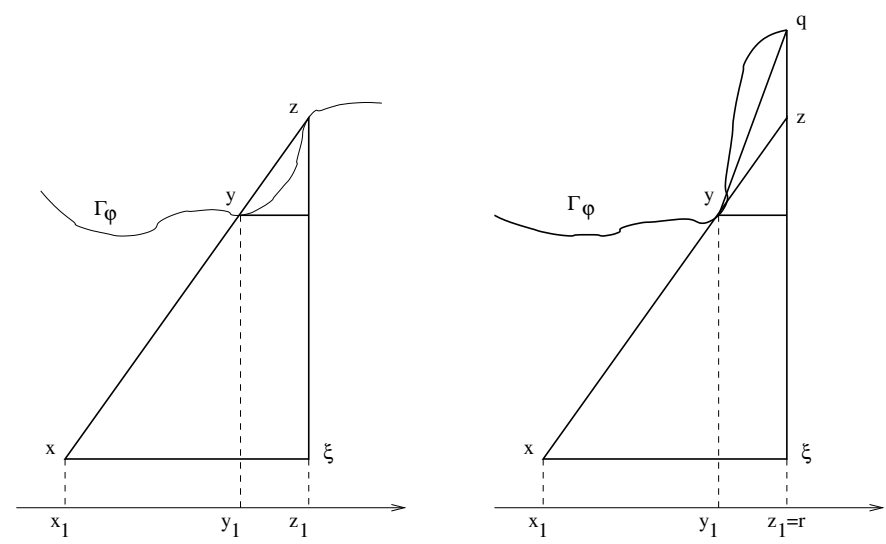

Fig. 3. Case i) at the left and case ii) at the right

Case i): $\mathbf{y}, \mathbf{z} \in \Gamma_{\varphi}$.

Let $\boldsymbol{\xi}=\left(\xi_{1}, \xi_{2}\right)$ be the point in the plane, such that the line passing throw $\boldsymbol{\xi}$ and $\mathbf{x}$ is parallel to the axis $O x_{1}$ and the line passing throw $\boldsymbol{\xi}$ and $\mathbf{z}$ is parallel to the axis $O x_{2}$.

We denote by $\alpha$ the angle $\widehat{\mathbf{x z} \boldsymbol{\xi}}$. Since $\mathbf{x} \in B_{R}(0)$ using (10), we get $|\mathbf{z}-\boldsymbol{\xi}| \geq$ $a-2 r$ and $|\mathbf{x}-\boldsymbol{\xi}| \leq 2 r$, then

$$
\tan \alpha=\frac{|\mathbf{x}-\boldsymbol{\xi}|}{|\mathbf{z}-\boldsymbol{\xi}|} \leq \frac{2 r}{a-2 r}
$$

By assumption we have $\mathbf{y}, \mathbf{z} \in \Gamma_{\varphi}$, then $\mathbf{y}=\left(y_{1}, a+\varphi\left(y_{1}\right)\right)$ and $\mathbf{z}=\left(z_{1}, a+\varphi\left(z_{1}\right)\right)$ with $-r<y_{1}, z_{1}<r$. We have also, $\tan \alpha=\frac{\left|y_{1}-z_{1}\right|}{\left|\varphi\left(y_{1}\right)-\varphi\left(z_{1}\right)\right|}$ and using (11) we get

$$
\tan \alpha \geq \frac{1}{L}
$$

This implies that

$$
\frac{2 r}{a-2 r} \geq \frac{1}{L}
$$

which is in contradiction with the hypothesis $L<\frac{a-2 r}{2 r}$. 
Case ii): $\mathbf{y} \in \Gamma_{\varphi}$ and $\mathbf{z} \in \partial \Omega_{\varphi} \backslash \Gamma_{\varphi}$.

As in the Case i), we construct $\boldsymbol{\xi}$, we denote by $\alpha$ the angle $\widehat{\mathbf{x z} \boldsymbol{\xi}}$ and we get

$$
\tan \alpha=\frac{|\mathbf{x}-\boldsymbol{\xi}|}{|\mathbf{z}-\boldsymbol{\xi}|} \leq \frac{2 r}{a-2 r} .
$$

Since $\mathbf{z}$ cannot belong to the bottom boundary of $C$, we assume that $\mathbf{z}$ belongs to the right boundary of $C$, so $\mathbf{z}=\left(r, z_{2}\right)$. We denote by $\mathbf{q}$ the point at the right top corner of $\Omega_{\varphi}$, so $\mathbf{q}=(r, a+\varphi(r))$. We have $W^{1, \infty}(-r, r) \subset \mathcal{C}^{0}([-r, r])$ (see [1], Theorem VIII.7, page 129), so $\varphi(r)$ is well defined.

Let $\alpha^{\prime}$ be the angle $\widehat{\mathbf{y q \xi}}$. Then

$$
\tan \alpha^{\prime}=\frac{\left|y_{1}-r\right|}{\left|\varphi\left(y_{1}\right)-\varphi(r)\right|} \geq \frac{1}{L} .
$$

If $z_{2}>a+\varphi(r)$ it follows that $\mathbf{z}$ belongs to the exterior of $\bar{\Omega}_{\varphi}$ not to $\partial \Omega_{\varphi}$, so $z_{2} \leq a+\varphi(r)$. In this case $\alpha \geq \alpha^{\prime}$ and we get

$$
\frac{1}{L}=\tan \alpha^{\prime}<\tan \alpha \leq \frac{2 r}{a-2 r}
$$

which is in contradiction with the hypothesis $L<\frac{a-2 r}{2 r}$.

Proposition 2. Let $\zeta:[-r, r] \rightarrow \mathbb{R}$ be a Lipschitz function of constant L, i.e.

$$
\forall x, y \in[-r, r], \quad|\zeta(x)-\zeta(y)| \leq L|x-y| .
$$

Denote by $\Gamma_{\zeta}=\left\{(x, \zeta(x)) \in \mathbb{R}^{2}, x \in[-r, r]\right\}$, the graph of $\zeta$.

Let $\mathbf{u}=\left(u_{1}, u_{2}\right): \Gamma_{\zeta} \rightarrow \mathbb{R}^{2}$ be such that

$$
\begin{aligned}
& \exists \eta_{1} \in\left(0, \frac{r}{2}\right), \quad \sup _{\mathbf{x} \in \Gamma_{\zeta}}|\mathbf{u}(\mathbf{x})| \leq \eta_{1} \\
& \exists \eta_{2} \in\left(0, \frac{1}{\sqrt{1+L^{2}}}\right), \forall \mathbf{x}, \mathbf{y} \in \Gamma_{\zeta}, \quad|\mathbf{u}(\mathbf{x})-\mathbf{u}(\mathbf{y})| \leq \eta_{2}|\mathbf{x}-\mathbf{y}| .
\end{aligned}
$$

Then there exits a Lipschitz function $\varphi:\left(-\frac{r}{2}, \frac{r}{2}\right) \rightarrow \mathbb{R}$ of constant less than $\frac{L+\eta_{2} \sqrt{1+L^{2}}}{1-\eta_{2} \sqrt{1+L^{2}}}$, such that its graph is included in $(\mathbf{I d}+\mathbf{u})\left(\Gamma_{\zeta}\right)$. If $\zeta(0)=0$, then

$$
\forall z \in\left(-\frac{r}{2}, \frac{r}{2}\right), \quad|\varphi(z)| \leq r\left(\sqrt{1+L^{2}}+\frac{1}{2}\right) .
$$

Proof. Let us introduce the function

$$
\phi:[-r, r] \rightarrow \mathbb{R}, \quad \phi(x)=x+u_{1}(x, \zeta(x)) .
$$

We will prove that $\phi$ is strictly increasing, so it is injective. To simplify, we assume that $\zeta$ and $\mathbf{u}$ are of class $\mathcal{C}^{1}$. We get

$$
\phi^{\prime}(x)=1+\frac{\partial u_{1}}{\partial x_{1}}(x, \zeta(x))+\frac{\partial u_{1}}{\partial x_{2}}(x, \zeta(x)) \zeta^{\prime}(x) .
$$


Since $\zeta$ is Lipschitz function of constant $L,\left|\zeta^{\prime}(x)\right| \leq L$. Similary, we have $|\nabla \mathbf{u}(\mathbf{x})| \leq \eta_{2}$. Using Cauchy-Schwarz we get

$$
\begin{aligned}
\left|\frac{\partial u_{1}}{\partial x_{1}}(x, \zeta(x))+\frac{\partial u_{1}}{\partial x_{2}}(x, \zeta(x)) \zeta^{\prime}(x)\right| & \leq \sqrt{\left(\frac{\partial u_{1}}{\partial x_{1}}\right)^{2}+\left(\frac{\partial u_{1}}{\partial x_{2}}\right)^{2}} \sqrt{1+L^{2}} \\
& \leq \eta_{2} \sqrt{1+L^{2}}
\end{aligned}
$$

It follows that

$$
\phi^{\prime}(x) \geq 1-\eta_{2} \sqrt{1+L^{2}}>0
$$

and then $\phi$ is strictly increasing, so $\phi$ is a bijection from $[-r, r]$ to $[\phi(-r), \phi(r)]$. From (13), we obtain $\phi(-r)<-\frac{r}{2}$ and $\phi(r)>\frac{r}{2}$.

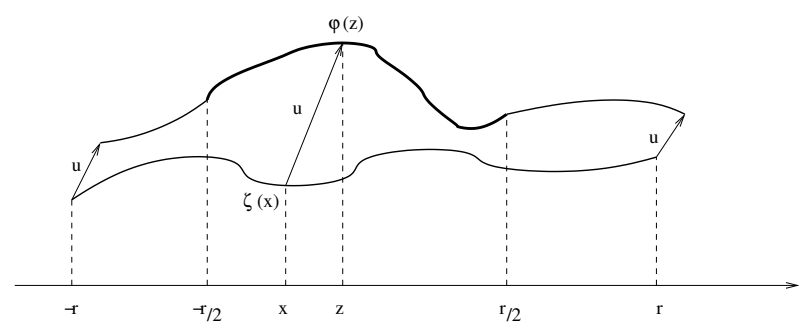

Fig. 4. The graphs of $\zeta$ and $\varphi$.

Let us introduce the function $\varphi:\left(-\frac{r}{2}, \frac{r}{2}\right) \rightarrow \mathbb{R}$,

$$
\varphi(z)=\zeta(x)+u_{2}(x, \zeta(x))
$$

where $x=\phi^{-1}(z)$. The function is well defined because if $z$ is in $\left(-\frac{r}{2}, \frac{r}{2}\right)$, then $x=\phi^{-1}(z)$ is in $(-r, r)$.

We will prove that $\varphi$ is a Lipschitz function. Let $z, w \in\left(-\frac{r}{2}, \frac{r}{2}\right)$ and $x=$ $\phi^{-1}(z), y=\phi^{-1}(w)$.

$$
\begin{aligned}
|\varphi(z)-\varphi(w)| & =\left|\zeta(x)-\zeta(y)+u_{2}(x, \zeta(x))-u_{2}(y, \zeta(y))\right| \\
& \leq|\zeta(x)-\zeta(y)|+\left|u_{2}(x, \zeta(x))-u_{2}(y, \zeta(y))\right| \\
& \leq L|x-y|+\eta_{2}|(x, \zeta(x))-(y, \zeta(y))| \\
& =L|x-y|+\eta_{2} \sqrt{(x-y)^{2}+(\zeta(x)-\zeta(y))^{2}} \\
& \leq\left(L+\eta_{2} \sqrt{1+L^{2}}\right)|x-y| .
\end{aligned}
$$


We have

$$
\begin{aligned}
|z-w| & =\left|x-y+u_{1}(x, \zeta(x))-u_{1}(y, \zeta(y))\right| \\
& \geq|x-y|-\left|u_{1}(x, \zeta(x))-u_{1}(y, \zeta(y))\right| \\
& \geq|x-y|-\eta_{2}|(x, \zeta(x))-(y, \zeta(y))| \\
& \geq|x-y|-\eta_{2} \sqrt{(x-y)^{2}+(\zeta(x)-\zeta(y))^{2}} \\
& \geq\left(1-\eta_{2} \sqrt{1+L^{2}}\right)|x-y| .
\end{aligned}
$$

We deduce that for all $z, w \in\left(-\frac{r}{2}, \frac{r}{2}\right)$

$$
\frac{|\varphi(z)-\varphi(w)|}{|z-w|} \leq \frac{\left(L+\eta_{2} \sqrt{1+L^{2}}\right)}{\left(1-\eta_{2} \sqrt{1+L^{2}}\right)}
$$

Let $(z, \varphi(z))$ be a point on the graph of $\varphi$, where $z \in\left(-\frac{r}{2}, \frac{r}{2}\right)$. Then there exits $x=\phi^{-1}(z) \in(-r, r)$. From the definition of $\phi$ and $\varphi$, we have

$$
\begin{aligned}
(z, \varphi(z)) & =\left(x+u_{1}(x, \zeta(x)), \zeta(x)+u_{2}(x, \zeta(x))\right) \\
& =(x, \zeta(x))+\left(u_{1}(x, \zeta(x)), u_{2}(x, \zeta(x))\right) \\
& =(\mathbf{I d}+\mathbf{u})(x, \zeta(x))
\end{aligned}
$$

which proves that the graph of $\varphi$ is included in $(\mathbf{I d}+\mathbf{u})\left(\Gamma_{\zeta}\right)$. Also

$$
\begin{aligned}
|\varphi(z)| & \leq|(z, \varphi(z))| \\
& =\left|\left(x+u_{1}(x, \zeta(x)), \zeta(x)+u_{2}(x, \zeta(x))\right)\right| \\
& \leq|(x, \zeta(x))|+\left|\left(u_{1}(x, \zeta(x)), u_{2}(x, \zeta(x))\right)\right| \\
& \leq \sqrt{x^{2}+(\zeta(x))^{2}}+\eta_{1} \leq \sqrt{r^{2}+(\zeta(x))^{2}}+\frac{r}{2}
\end{aligned}
$$

If $\zeta(0)=0$, since $\zeta$ is a Lipschitz function of constant $L$, we have

$$
|\zeta(x)|=|\zeta(x)-\zeta(0)| \leq L|x-0|<L r .
$$

Then $|\varphi(z)| \leq r\left(\sqrt{1+L^{2}}+\frac{1}{2}\right)$.

\section{Uniform Estimation of the Radius of the Ball for Small Perturbation of Lipschitz Domain}

Definition 1 (see [2]). Let $r, a, L$ be three positive numbers and $\Omega$ an open bounded set of $\mathbb{R}^{2}$. We say that the boundary $\partial \Omega$ is uniform Lipschitz if, for every $\mathbf{x}_{0} \in \partial \Omega$, there exits a Cartezian coordinates system $\left\{x_{1}, x_{2}\right\}$ of origin $\mathbf{x}_{0}$ and a Lipschitz function $\zeta:(-r, r) \rightarrow(-a, a)$ of constant $L$, such that $\zeta(0)=0$,

$$
\begin{aligned}
\partial \Omega \cap P\left(\mathbf{x}_{0}\right) & =\left\{\left(x_{1}, \zeta\left(x_{1}\right)\right), x_{1} \in(-r, r)\right\}, \\
\Omega \cap P\left(\mathbf{x}_{0}\right) & =\left\{\left(x_{1}, x_{2}\right), x_{1} \in(-r, r), \zeta\left(x_{1}\right)<x_{2}<a\right\},
\end{aligned}
$$

where $P\left(\mathbf{x}_{0}\right)=(-r, r) \times(-a, a)$. 
We will use the same notation as in the first section. We will prove a result similar to the Lemma 3.2 , p. 40, from [3], but the radius of the balls is the same for all admissible displacements.

Theorem 1. Let $\partial \Omega_{0}^{S}$ be an uniform Lipschitz boundary of parameters $r, a, L$. We set $R \in\left(0, \frac{r}{2}\right)$. There exists two constants $\eta_{1} \in\left(0, \frac{r}{2}\right), \eta_{2} \in\left(0, \frac{1}{\sqrt{1+L^{2}}}\right)$ and two natural numbers $m, n \in \mathbb{N}^{*}$, such that for all $\mathbf{u}: \partial \Omega_{0}^{S} \rightarrow \mathbb{R}^{2}$, such that

$$
\begin{aligned}
& \sup _{\mathbf{x} \in \partial \Omega_{0}^{S}}|\mathbf{u}(\mathbf{x})| \leq \eta_{1} \\
& \forall \mathbf{x}, \mathbf{y} \in \partial \Omega_{0}^{S}, \quad|\mathbf{u}(\mathbf{x})-\mathbf{u}(\mathbf{y})| \leq \eta_{2}|\mathbf{x}-\mathbf{y}|
\end{aligned}
$$

we have the decomposition

$$
\Omega_{u}^{F}=\left(\bigcup_{i=1}^{m} \Omega_{i}\right) \bigcup\left(\bigcup_{j=m+1}^{m+n} B_{\eta_{1}}\left(\mathbf{x}_{j}\right)\right)
$$

where $\Omega_{i}=\Omega_{u}^{F} \cap Q\left(\mathbf{x}_{i}\right)$ for $1 \leq i \leq m$ are star-shaped domains with respect to every point of a ball $B_{i}$ of radius $R, \bar{B}_{i} \subset \Omega_{i}, Q\left(\mathbf{x}_{i}\right)$ are rectangles of center $\mathbf{x}_{i} \in \partial \Omega_{0}^{S}$ congruent to $\left(-\frac{r}{2}, \frac{r}{2}\right) \times(-a, a)$ and $B_{\eta_{1}}\left(\mathbf{x}_{j}\right)$ for $m+1 \leq j \leq m+n$ are balls of center $\mathbf{x}_{j} \in \Omega_{u}^{F}$ and radius $\eta_{1}, \bar{B}_{\eta_{1}}\left(\mathbf{x}_{j}\right) \subset \Omega_{u}^{F}$.

Proof. Let us remark that if $\Omega_{0}^{S}$ is Lipschitz of parameters $r, a, L$, then it is also for the parameters $r^{\prime}, a, L$, for $r^{\prime}<r$. Consequently, we can choose $r$ small enough such that $2 L+\sqrt{1+L^{2}}+\frac{5}{2}<\frac{a}{r}$.

Let $P(\mathbf{x})$ be the rectangle of center $\mathbf{x} \in \partial \Omega_{0}^{S}$ given by the Definition 1 . We denote by $Q(\mathbf{x})$ the rectangle $\left(-\frac{r}{2}, \frac{r}{2}\right) \times(-a, a)$ using the same local coordinates. We have $\partial \Omega_{0}^{S} \subset \cup_{\mathbf{x} \in \partial \Omega_{0}^{S}} Q(\mathbf{x})$ and since $\partial \Omega_{0}^{S}$ is compact, then there exits $m \in \mathbb{N}^{*}$ and $\mathbf{x}_{i} \in \partial \Omega_{0}^{S}, 1 \leq i \leq m$, such that

$$
\partial \Omega_{0}^{S} \subset \bigcup_{i=1}^{m} Q\left(\mathbf{x}_{i}\right)
$$

We will use the notation

$$
\begin{aligned}
\mathcal{O}_{\eta} & =\left\{\mathbf{x} \in \mathbb{R}^{2} ; d\left(\mathbf{x}, \partial \Omega_{0}^{S}\right) \leq \eta\right\} \\
\Omega_{0, \eta}^{F} & =\left\{\mathbf{x} \in \Omega_{0}^{F} ; d\left(\mathbf{x}, \partial \Omega_{0}^{S}\right)>\eta\right\}
\end{aligned}
$$

where $d(\mathbf{x}, A)=\inf _{\mathbf{y} \in A}|\mathbf{x}-\mathbf{y}|$ is the distance function. There exists $\eta_{0}>0$, such that

$$
\mathcal{O}_{\eta_{0}} \subset \bigcup_{i=1}^{m} Q\left(\mathbf{x}_{i}\right)
$$

Set $\eta_{1}=\min \left(\frac{\eta_{0}}{2}, \frac{r}{2}\right)$. 
Let $\mathbf{u}: \partial \Omega_{0}^{S} \rightarrow \mathbb{R}^{2}$ be such that (15) holds. Then $(\mathbf{I d}+\mathbf{u})\left(\partial \Omega_{0}^{S}\right) \subset \mathcal{O}_{\eta_{1}} \subset \mathcal{O}_{\eta_{0}}$, for all admissible $\mathbf{u}$ and it follows that

$$
\Omega_{u}^{F} \subset \mathcal{O}_{\eta_{0}} \cup \Omega_{0, \eta_{0}}^{F}
$$

Since $\Omega_{0, \eta_{0}}^{F}$ is bounded, then there exists $n \in \mathbb{N}^{*}$ and $\mathbf{x}_{j} \in \Omega_{0, \eta_{0}}^{F}$ for $m+1 \leq j \leq$ $m+n$, such that

$$
\Omega_{0, \eta_{0}}^{F} \subset \bigcup_{j=m+1}^{m+n} B_{\eta_{1}}\left(\mathbf{x}_{j}\right)
$$

But $\eta_{1} \leq \frac{\eta_{0}}{2}$, so $\bar{B}_{\eta_{1}}\left(\mathbf{x}_{j}\right) \subset \Omega_{u}^{F}$. From (17), (18), (19), we get

$$
\Omega_{u}^{F}=\left(\bigcup_{i=1}^{m}\left(\Omega_{u}^{F} \cap Q\left(\mathbf{x}_{i}\right)\right)\right) \bigcup\left(\bigcup_{j=m+1}^{m+n} B_{\eta_{1}}\left(\mathbf{x}_{j}\right)\right) .
$$

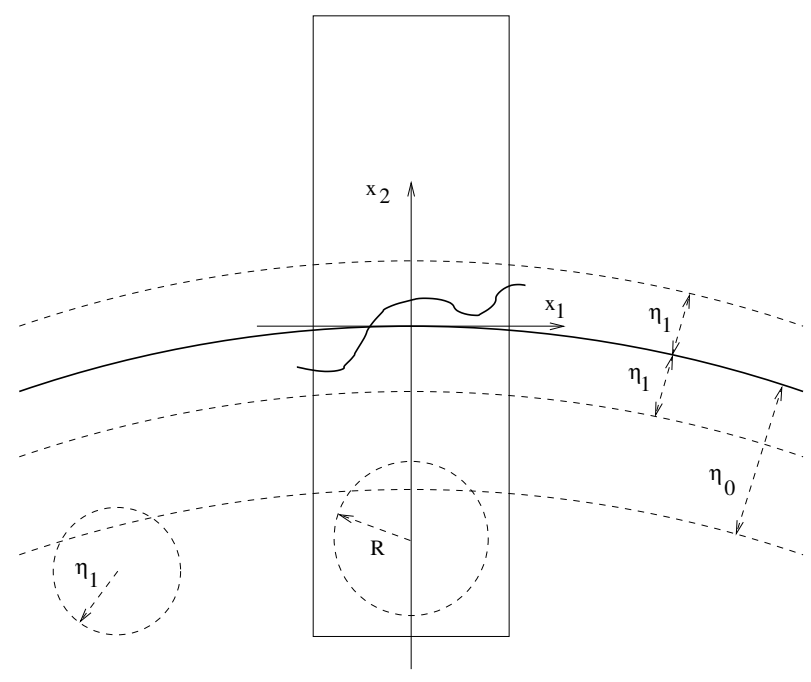

Fig. 5. The rectangle $\left(-\frac{r}{2}, \frac{r}{2}\right) \times(-a, a)$ is of center $\mathbf{x}_{i} \in \partial \Omega_{0}^{S}$, the ball of radius $\eta_{1}$ is of center $\mathbf{x}_{j} \in \Omega_{0, \eta_{0}}^{F}$.

Using the Proposition 2, $(\mathbf{I d}+\mathbf{u})\left(\partial \Omega_{0}^{S}\right) \cap Q\left(\mathbf{x}_{i}\right)$ is the graph of a Lipschitz function $\varphi:\left(-\frac{r}{2}, \frac{r}{2}\right) \rightarrow(-a, a)$ of constant

$$
L^{\prime}=\frac{L+\eta_{2} \sqrt{1+L^{2}}}{1-\eta_{2} \sqrt{1+L^{2}}}
$$

and $|\varphi| \leq r\left(\sqrt{1+L^{2}}+\frac{1}{2}\right)$. If $\eta_{2} \in\left[0, \frac{1}{2 \sqrt{1+L^{2}}}\right)$, then $L^{\prime} \in[L, 2 L+1)$. 
We have $2 L+1<\frac{a-r\left(\sqrt{1+L^{2}}+\frac{3}{2}\right)}{r}$ by the choice of $r$ at the begining of the proof. Using a similar argument as in the Proposition 1, we get that $\Omega_{i}=$ $\Omega_{u}^{F} \cap Q\left(\mathbf{x}_{i}\right)$ is a star-shaped domain with respect to every point of the ball of radius $R$ and center $\left(0,-a+\frac{r}{2}\right)$.

Corollary 1. Using the Theorem 3.1, p. 129, from [3], we get that the constant $K_{1}$ from the inequality (7) depends on $\min \left(\eta_{1}, R\right)$ and diam $(D)$, but it is independent on the displacement $\mathbf{u}$.

\section{References}

1. Brezis, H.: Analyse fonctionnelle : théorie et applications. Dunod, Paris, 2005.

2. Chenais, D.: On the existence of a solution in a domain identification problem. J. Math. Anal. Appl. 52 no. 2, 189-219, (1975).

3. Galdi, G.: An introduction to the mathematical theory of the Navier-Stokes equations. Vol. I. Linearized steady problems. Springer Tracts in Natural Philosophy, 38. Springer-Verlag, New York, 1994.

4. Halanay, A., Murea, C.M., Tiba, D.: Existence and approximation for a steady fluid-structure interaction problem using fictitious domain approach with penalization, Mathematics and its Applications 5, no. 1-2, 120-147, (2013).

5. Halanay, A., Murea, C.M., Tiba, D.: Existence of a steady flow of Stokes fluid past a linear elastic structure using fictitious domain, submitted 